Página inicial: 217 - Página final: 240

TIPO DE ARTÍCULO: de Investigación

\title{
REPARACIÓN A LAS VÍCTIMAS DE DICTADURAS, CONFLICTOS ARMADOS Y VIOLENCIA POLÍTICA EN SUS COMPONENTE DE COMPENSACIÓN, SATISFACCIÓN, REHABILITACIÓN Y NO REPETICIÓN. ${ }^{4}$
}

\author{
REPARATION FOR VICTIMS OF DICTATORSHIPS, ARMED CONFLICTS AND POLITICAL \\ VIOLENCE IN THEIR COMPONENTS OF COMPENSATION, SATISFACTION, REHABILITATION, \\ AND NON-REPETITION.
}

\begin{tabular}{|c|}
\hline Recibido Revisado Aceptado \\
\hline
\end{tabular}

Por: Juan David Villa1, Daniela Londoño Diaz², Daniela Barrera Machado. ${ }^{3}$

\section{RESUMEN:}

El presente texto segundo de dos entregas, realiza un acercamiento a las investigaciones realizadas en la pasada década y a comienzos de la presente sobre los procesos de reparación, en el marco de procesos de justicia transicional en países en procesos que van de la guerra a la paz y de la dictadura a la democracia. Se revisan investigaciones que versan sobre las consecuencias de los procesos de reparación y de transición en las víctimas, en la reconstrucción del tejido social, en la generación de procesos de reconciliación y la transformación del sujeto individual y colectivo.

\section{PALABRAS CLAVES:}

Víctimas, Reparación, Atención psicosocial, Justicia Transicional

\section{ABSTRACT:}

This current text, which is the second of two issues, carries out an approach of investigations conducted in the past decade and at the beginning of the present decade on the reparation processes, within the context of transitional justice processes, in countries which are on the way to peace, and from dictatorship to democracy. We review research dealing with the consequences of the reparation and transition processes of victims, in the reconstruction of the social fabric, in the generation of reconciliation processes, and the transformation of the individual and collective subject.

\section{Key words:}

Victims, Reparation, Psychosocial Attention, and Transitional Justice Introducción.

${ }^{1}$ Psicólogo - Pontificia Universidad Javeriana, Magister y Doctor en Cooperación Internacional al Desarrollo Universidad Pontificia de Comillas. Docente investigador de la Facultad de Psicologia Universidad de San Buenaventura Medellín - Colombia. Contacto: Juand.villa@usbmed.edu.co.

${ }^{2}$ Joven investigadora, Universidad de San Buenaventura, Medellin, Colombia.

${ }^{3}$ Joven investigadora, Universidad de San Buenaventura, Medellín, Colombia 
Los trabajos revisados en la presente investigación referentes a la reparación, y más ampliamente a la justicia transicional, realizan importantes contribuciones para reflexionar y repensar las formas en que actualmente se están desarrollando estos mecanismos y procesos en Colombia, poniendo en evidencia algunas fallas y aciertos desde la perspectiva y el interés por el beneficio de las personas víctimas. Dichos trabajos proveen además recomendaciones para hacer de estos procesos más efectivos y menos revictimizantes, de tal forma que realmente se consiga el objetivo de promover la transición de la guerra a la paz, la reconciliación nacional y la recuperación de las víctimas.

Las investigaciones apuntan a que la complementariedad entre los mecanismos de verdad, justicia y reparación resulta ser un elemento fundamental para que las personas víctimas se sientan realmente reparadas y no revictimizadas. Sin embargo, en diversos países, como Colombia, las acciones de verdad y justicia han estado caracterizadas por fallas y restricciones en la voluntad, estructura y proceder institucional y político, por el silenciamiento de ciertas voces, por la impunidad, por la presión de discursos dominantes que promueven el olvido y por el mantenimiento del status quo, lo que ha generado más daños en la población víctima y ha impedido que ésta se sienta reparada.

Así mismo, cuando la reparación termina por centrarse única y exclusivamente en lo material, ocasiona efectos perniciosos en la población que la recibe, entre estos es posible señalar la disminución de la autoestima, de la confianza y de la esperanza y la aparición o el incremento de sentimientos de culpa que conduce a que se perciba el dinero recibido como una humillación o "dinero fácil", lo que exacerba los daños e impactos generados por los hechos victimizante y no dignifica. En el contexto colombiano estas afirmaciones adquieren materialidad, puesto que muchas personas víctimas significan la reparación como una caridad que no repara y se perciben a sí mismos como receptores de una "ayuda" del Estado sin lograr, antes de ésta o a través de ésta, reconocerse como sujetos de derechos, cuyo cumplimiento es un deber del Estado (Villa, 2013, 2014).

No puede negarse que la indemnización o compensación es fundamental dentro del proceso de reparación en Colombia, incluso Jaramillo Marín (2010) afirma que la apuesta ideal en términos de reparación en el país implica una convergencia entre medidas materiales y simbólicas. Ahora bien, la clave del proceso de reparación no está en lo que se haga: si se da una indemnización o se construye un monumento o se hace alguna restitución; la clave está en la forma: que la indemnización no sea una compra ni una transacción, que los monumentos no sean una forma de pasar la página rápido. Siempre, y en todo momento, la clave está en el reconocimiento público, social y moral que se haga de las víctimas (Hamber, 2000; Martín Beristain, 2008, 2010a, 2010b; Gómez Isa, 2008; Villa, 2013).

\section{Compensación:}

Este componente de la reparación "busca reparar a las víctimas por los daños materiales fisicos, mentales, a la reputación y a la dignidad sufridos, los gastos 
incurridos, las pérdidas de ingreso y de oportunidades, y los costos de asistencia juridica y servicios médicos, entre otros, cuando no sea posible la restitución a la situación anterior o cuando las victimas no la deseen" (Centro Internacional para la Justicia Transicional y Centro de Estudios de Derecho, Justicia y Sociedad , 2009, pág. 40). Como se ha anotado anteriormente, parece ser la medida que sigue primando en el contexto colombiano.

Muchos autores e investigadores como Hayner (2008a) consideran que el pago de indemnizaciones debe ser un requisito indispensable en los proceso de reparación, porque simbólicamente permite identificar que el Estado se hace cargo y asume un nivel de responsabilidad por lo sucedido. Y de hecho, como se puso de manifiesto en el apartado anterior y como afirman Gutiérrez Agüello et.al (2006) y Villa (2012, 2013, 2014), en Colombia los alcances legislativos y las acciones desarrolladas en torno al tema de la reparación se han centrado especialmente en la indemnización económica de las víctimas. De forma sintónica con estos resultados, la investigación transcultural realizada por Kiza, Rathgeber \& Rohne (2006) indica que, para la mayoría de víctimas que participaron de esta, la reparación monetaria seguida de la construcción de memoriales son las medidas, en términos de reparación, que cuentan con mayor apoyo a nivel estatal.

El trabajo de Jaramillo (2012) con las comunidades indígenas wayúu, que se trajo a colación anteriormente, permite observar precisamente cómo la compensación ha aparecido como la medida privilegiada y casi exclusiva que ofrece el Estado, pues en estas comunidades se observa una naturalización de la deuda que el Estado tiene con las víctimas del conflicto armado en Colombia, significando la relación entre ambas partes en términos monetarios, es decir, de compensación económica acogida bajo la premisa de reparación administrativa promovida por el Estado, lo que ha afectado significativamente en la identidad de esta comunidad. Sin embargo, el autor agrega que la organización indigena ha resistido esta propuesta, fomentando la construcción de nociones de reparación mucho más amplias, que abarcan el respeto y la compensación por el daño a la cultura y la violación a los derechos territoriales. Por esta razón la reparación de las personas víctimas debe ser más que dinero y deben abarcar un portafolio de justicia transicional, en el que se debe tener especial cuidado en la implementación de los programas. Es por ello que es necesario que se implementen medidas en múltiples sentidos.

A la luz de esta perspectiva, Mack (2007) cuestiona el plan de reparación en Guatemala (PNR) porque se centró en una compensación monetaria que a lo sumo ha servido para paliar niveles de precariedad económica, además de hacerse con lentitud; pero además, por el contraste marcado entre este apoyo y el que se ha dado a los exmilitantes de las PAC (Patrullas de autodefensa campesina. Forma de organización paramilitar, formadas y apoyadas por el ejército de Guatemala en lógica de lucha contrainsurgente. Tienen una alta cuota de responsabilidad en las violaciones de los derechos humanos en este país.), que ha sido más expedito. De forma similar a lo sucedido en Colombia en relación con la denominada desmovilización paramilitar, frente a la cual las víctimas sienten un desequilibrio 
en los niveles de atención y servicios ofrecidos por el Estado en términos de atención (Arévalo, 2010; Villa, 2012, 2013).

Viaene (2008), por su parte, también en el contexto guatemalteco, detecta desencuentros al aplicar un modelo occidentalizado a una cultura que tiene lengua, costumbres y una cosmovisión diferentes. Por otro lado, porque consideran que este plan debe reestructurarse totalmente en función de responder a las demandas y necesidades reales de las víctimas. Para los autores, las comunidades tienen una visión clara de su reparación, reconocen la importancia de la memoria histórica y finalmente la utilización de rituales locales de reparación. Pero el Estado desoye estas voces y plantea un programa al margen de estas, que además no se cumple, puesto que se sigue encubriendo y manteniendo en posiciones de poder, a muchos de los responsables de las violaciones de derechos humanos en el pasado.

Esto sugiere que el reto para el Estado no está sólo en reconocer y acoger las diferentes actitudes, percepciones o preferencias de las víctimas en los temas de verdad, justicia y reparación; que, como se mencionó en el apartado anterior, tienen un profundo anclaje en las características socioculturales y políticas en las que estas se encuentran y no sólo en aplicar dos o tres medidas de reparación juntas; sino que además dicho reto implica, fundamentalmente, que se dé una inclusión de las necesidades específicas y de las voces de estas víctimas dentro de la formulación de los programas y las medidas de reparación y dentro de la planeación de las estrategias para llevarlas a cabo y que se validen sus relatos, narrativas, experiencias, vivencias y emociones.

\section{Satisfacción.}

Este tipo de medidas "Buscan reconocer públicamente el daño sufrido por las víctimas y a través de ello dignificarlas, para lo cual incluyen, entre otras, la investigación y sanción de los responsables de los crimenes, la difusión de la verdad, la búsqueda de los desaparecidos y de los restos de los muertos, la solicitud pública de disculpas y la realización de conmemoraciones y homenajes a las victimas" (Centro Internacional para la Justicia Transicional y Centro de Estudios de Derecho, Justicia y Sociedad , 2009, pág. 40). Por su parte, Martín Beristain \& Pérez Sales (2008) sostienen que:

"Las medidas simbólicas, asi como las formas en que se expresa la memoria colectiva sobre las violaciones a los derechos humanos son huellas que marcan el camino de asumir la verdad, proporcionan apoyo a las víctimas y suponen una ruptura con los perpetradores. Contribuyen a un cambio de clima social cuando responden a las demandas de las víctimas y no son una simple fachada (...)” (pág. 33)

Siguiendo la línea de sentido de estas definiciones y de algunos aspectos esbozados en apartados anteriores, la revisión de diferentes investigaciones indican que los actos de memoria son de gran envergadura para las víctimas dentro de los procesos de reparación en el marco de justicia transicional (Lira, 2010; Jaramillo Marín, 2011 b; Molina Valencia, 2010; Villa, 2012, 2013). Sobre el curso de estas ideas, Lira (2010), partiendo de la experiencia chilena, destaca el papel de la memoria 
en los procesos de reparación, señalando que el trabajo sobre este aspecto debe constituir uno de los esfuerzos desarrollados por las autoridades para reparar a las víctimas ya que esta convoca a la no repetición.

La reconstrucción de la memoria impacta positivamente en la salud mental de las sociedades que han vivido bajo la sombra de la violencia, permitiendo cerrar las heridas que han quedado abiertas luego de años de olvido y reforzar la cohesión social. Sin embargo, es un proceso complejo, continuo y abierto que implica una constante reconciliación de versiones distintas de un mismo evento, que construye un puente entre el presente, pasado y futuro y promueve el empoderamiento de los sujetos, familias y comunidades (Gaborit, 2006; Jaramillo Marín, 2010; Villa, 2012, 2013). La reconciliación de versiones supone un reto de inclusión, pues demanda que aquellas experiencias de vida y resistencia que históricamente han sido excluidas comiencen a ser contadas y tenidas en cuenta en las memorias colectivas (Pinto Velásquez, 2011).

Algunos autores como Cortés Severino (2007) y Villa (2012) plantean que la memoria debe incluir las historias afectivas de la población, visibilizando así el sufrimiento de la gente, recogiendo su dignidad y sus resistencias, además de sus voces encarnadas en actos culturales y corporales, no ubicados necesariamente en la lógica racional del hecho, fechas y archivos. Con lo cual se va reconstruyendo el tejido social y se devuelve dignidad a la gente, a las poblaciones, a sus sentidos de vida.

En consonancia con estas ideas, Marques, Páez y Serra (1998) afirman que, a través de acciones como las conmemoraciones testimoniales, puede generarse una resignificación positiva de los hechos; pero también pueden traer recuerdos intrusivos individuales. Sin embargo, cuando hay un reconocimiento del hecho, de su injusticia y de que no debería pasar jamás, esto suele llevar a dicha resignificación positiva (Jodelet, 1998).

Llegado este punto resulta importante anotar que muchas de las iniciativas de actos de memoria, emparejadas a sus efectos benéficos en los procesos de recuperación emocional, moral, sociocultural y politico, han surgido al interior de las mismas comunidades, expresadas por medio de sus rituales tradicionales o de rituales creados con aras de hacer frente a los daños e impactos generados por la violencia (Uribe, 2010; Villa Gómez, 2012, 2013). De hecho, en el caso colombiano, después de iniciada la implementación de la Ley de Justicia y Paz comenzaron a emerger unos discursos emanados de algunos agentes gubernamentales y de unos actores desmovilizados que promovian más la reconciliación que la justicia y en corriente con ello, promovian más el "derecho al olvido" que el "deber de recordar"; frente a esto aparecieron, y siguen apareciendo, narrativas humanitarias que fueron potenciadas por las mismas organizaciones de víctimas, y que demandan acciones y políticas de memoria frente a las politicas de olvido (Jaramillo Marín, 2010b)

Para ilustrar las acciones de memoria realizadas desdey por las mismas comunidades, resulta importante traer a escena la experiencia de Martha Nubia Bello $(2005,2007)$ quien realizó un trabajo con la comunidad de Bojayá en el Chocó, Colombia; donde, 
a partir de rituales de purificación, bordados de un manto con los nombres de las víctimas, jornadas de duelo, se dieron pasos hacia el restablecimiento de vínculos comunitarios y la reconstrucción del tejido social: "Estas prácticas muestran que de manera autónoma y con sus propios recursos, la comunidad recuerda y procesa su pasado doloroso y puede expresar sus sentimientos" (Bello, 2005, p. 16). Es por esto que la autora afirma que el daño y la manera de afrontarlo no se pueden establecer a priori, según estándares universales, sino que hay que atender la complejidad del entramado de relaciones de cada espacio concreto.

Cabe destacar también la experiencia de las tejedoras de Mampuján en el norte de Bolivar, Colombia, y de las mujeres narradoras de historias en los Montes de Maria, las cuales se han organizado en torno a la recuperación de su propia memoria, pero que al mismo tiempo han posibilitado espacios de contención, apoyo, elaboración del duelo y reconstrucción del tejido social (Uribe, 2010).

La experiencia de Gasparre, Bosco y Bellelli (2010) en su investigación desarrollada en Guatemala, es también sugerente acerca del tema. Estos autores mostraron que la participación de las víctimas en rituales, reuniones para celebrar y recordar y la participación en acciones de movilización generaban mayor apoyo social, conductas altruistas, afrontamiento comunitario, compromiso en acciones políticas y del movimiento de derechos humanos. Además la participación en estas acciones públicas de memoria se asoció con crecimiento postraumático, que implicaba, según Janoff-Bulman (2004) una mejor concepción del sí mismo, de los otros y de la sociedad.

Hamber, Mosikare, Friedman \& Maepa (2000), Hamber (2003) Lykes, Blanche \& Hamber (2003) y Hayner (2008a) reportan el proceso de Khulumani, organización de víctimas que, entre otras labores, desarrolló rituales con las comunidades y con las victimas en varias localidades en Sudáfrica. Se trataba de vigilias de luces para recordar a los seres queridos asesinados. Estos autores mostraron que este proceso trajo consolación, soporte emocional y cohesión colectiva, puesto que la gente pudo expresarse colectivamente y romper el silencio del pasado. Procesos similares pueden reportarse en Colombia (Villa et al. 2007) y en Guatemala (Viane, 2010)

Igreja (2007) Igreja, Dias-Lambranca \& Richters (2008) y Viaene (2008) presentan la experiencia de la comunidad de Gorongosa, en Mozambique, que posibilitó procesos de recuperación individual (puesto que permitió la elaboración de emociones como la rabia, el dolor, el miedo y el odio) y reconstrucción del tejido colectivo, en rituales, que permitieron la liberación de mujeres que se sentian subjetivamente poseídas por los espiritus 'Gamba", al parecer estos espiritus representaban la culpa familiar y el dolor comunitario y tomaban posesión de mujeres, familiares de perpetradores, y no les abandonaban hasta que éstos, los perpetradores, no hubieran señalado y nombrado todos sus crimenes; con lo cual se tenía que hacer un ritual de purificación colectivo. El acusado debía admitir su responsabilidad y participar del ritual colectivo asumiendo su acción, con lo cual el espíritu abandona a la mujer atormentada, abriendo un escenario de justicia y reconciliación en un marco familiar y comunitario. Los autores piensan que son formas de transformación social 
más allá de la simple narrativa, pero que trasciende la memoria y el olvido. Por lo que concluyen que los dispositivos más occidentales de la psicologia, las ciencias médicas y las ciencias jurídicas deben estar abiertos a dialogar con estas formas tradicionales; puesto que son caminos válidos para trabajar en la reconstrucción del tejido social, la sanación de las víctimas, la reconciliación, la construcción de la paz y la aplicación de formas de justicia transicional (Cfr. Hayner , 2008a; Bello \& Millan, 2005; Estrada Mesa, Ripoll Núñez, \& Rodríguez Charry, 2010; Rebolledo \& Rondón, 2010).

Otros autores como Bowlby (1980), Worden (1991, 1997), Fernández Liria (2006), Martín Beristain (2005), han señalado que los ritos funerarios y las manifestaciones colectivas de duelo tienen un potencial para ayudar a aceptar la pérdida, para poner el limite entre los que han muerto y los que quedan vivos. Posibilitan una catarsis emocional y una oportunidad de manifestación abierta del dolor, la tristeza y la rabia. Por esta razón, se ha afirmado que tienden a fortalecer la cohesión colectiva, mientras no son protectores frente a las emociones negativas. Por lo tanto, será importante diferenciar entre los rituales de duelo y funerarios, de las acciones políticas y públicas de memoria en el marco social, puesto que sus intencionalidades y procesos son diferentes.

Por otro lado, Hamber (2000) afirma, por su experiencia con grupos de víctimas en Sudáfrica, que en ausencia de reparación, de justicia y en un escenario donde las verdades todavía son incompletas, es fundamental realizar un trabajo desde abajo, fortaleciendo las comunidades, haciendo apoyo mutuo, construyendo simbolos, monumentos, realizando rituales y movilizaciones para que la dignificación venga, por lo menos desde la comunidad, y la recuperación emocional de las víctimas no dependa de los juegos de poder político; lo que también sería válido y necesario para el contexto Colombiano.

Estas afirmaciones han sido apoyadas por Conejero, De Rivera, Páez \& Jiménez (2004). Campos \& Páez (2004), Páez, Basabé, Cubillas \& González-Castro (2007) y Rimé, Páez, Basabe \& Martínez (2009), quienes demuestran que el compartir emocional y la participación en los eventos (rituales religiosos, acciones políticas, marcha de protesta), pueden convertirse en una forma de afrontamiento que posibilita un mejoramiento en el clima emocional. También la participación en rituales favorece la cohesión social y restablecimiento del vínculo social que fortalece la identidad colectiva, permite alcanzar altos niveles de integración social con disminución de la sensación de soledad social y aumento del vínculo social, mejor estado de ánimo; disminución de la sensación de impotencia y de la ansiedad y reconstrucción de creencias sociales positivas contribuyendo con esto a una experiencia de afrontamiento colectivo y a la generación de cultura de paz y una alta percepción de crecimiento postraumático en un clima social positivo. Aunque también coinciden en que no se transforman las emociones negativas de dolor, tristeza y rabia.

Puede decirse entonces que las acciones rituales, públicas y performativas, tienen una dimensión terapéutica (Beristain, Páez, Rimé, Kanyangara, 2010): Las arpilleras 
en Chile (Lira, 1998; Bacic, 2008), los escraches (Los escraches son acciones noviolentas de carácter lúdico y pedagógico que buscan identificar y señalar a personas, instituciones y otros responsables de los crimenes cometidos contra la dictadura. Es una forma de acción social que han desplegado los movimientos sociales en Argentina, como mecanismo para luchar contra la impunidad y generar algún tipo de sanción social, ya que las sanciones del Estado, o bien no se dan, o bien tardan demasiado en llegar) en Argentina (Oberti, 2006; Daleo, 2007; Pastoriza, 2009; Sarlo, 2009), las múltiples acciones artísticas de los jóvenes, las madres y otros sectores sociales en Argentina: grafitis, murgas, conciertos de rock, obras de teatro (Rojas y Canevese, 2000, Correa, 2002; Molas y Molas, 2006); las expresiones culturales desarrolladas en Colombia: telones, murales, jornadas culturales, fotografias, marchas simbólicas, arreglo de cementerios, en el Oriente Antioqueño y en el resto del país (Villa et. al. 2007; Uribe, 2008, 2009, 2010), el trabajo con fotografias en Guatemala, las fiestas mayas, la cruz en el cerro del Filo o en Xelabé, la multiplicidad de experiencias locales después de los acuerdos de paz, el proceso de elaboración del REMHI (REMHI: informe de la oficina de derechos humanos del arzobispado de Guatemala sobre recuperación de la memoria histórica, que implicó un proceso de formación, acompañamiento y trabajo psicosocial con víctimas por todo el país.), la conmemoración anual de la masacre de Xamán (Lykes, 2001 a, b; Martin Beristain, 1999, 2003, 2006, 2007; Cabrera, 2004; Viaene, 2008), tienen consecuencias, no solamente en la recuperación de los individuos, sino también en la reconstrucción del tejido social.

Para Elizabeth Lira (1998), tanto la memoria documentada, como la memoria social performativa (memoria viviente), contribuyen al restablecimiento o la construcción de un orden político justo que genere un marco ético, político y subjetivo para enfrentar los abusos del pasado. Por eso a nombre de evitar el dolor o evitar la polarización o que no se den venganzas y/o violencia, no se puede eliminar la memoria. El arte y el performance cumplen un papel importante, porque la gente necesita que sus relatos sean reconocidos por la sociedad, por otros fuera de ellos mismos, para hacer que su horror privado sea parte de la historia, parte de lo que otros reconocen como verdadero, válido y compartido; además, para contribuir a la transformación de las condiciones actuales, haciendo posible que emerjan nuevos campos de sentido que difieren de las versiones hegemónicas, que en múltiples ocasiones atentan contra la dignidad de las personas victimas (Piper, Fernández, Íñiguez, 2013).

Se hace entonces evidente que los procesos de reparación por los que han atravesado comunidades en diferentes lugares del mundo son complejos y no se agotan en las acciones institucionales, sino que las iniciativas y acciones de la misma gente juegan también un papel crucial para la recuperación emocional, moral, sociocultural y política. De ahí la importancia que el Estado reconozca, valide y acoja estas prácticas.

Además de los actos de memoria y los rituales de las comunidades, diversas experiencias han mostrado que la petición de disculpas resulta ser también un proceso reparador para las víctimas. En Bélgica, Lastrego (2009) y Lastrego \& Licata 
(2010) realizaron una investigación cuantitativa en la que mostraron que la petición de disculpas del primer ministro belga ante el pasado colonial, habia generado mejor clima emocional en el grupo de estudio, en comparación con el grupo control, donde sólo se recordaban los hechos dolorosos de la colonización. Y Valencia (2010) mostró que la petición oficial de disculpas refuerza la cohesión social y el clima emocional; puesto que permite una percepción de solidaridad y confianza social; lo cual se hizo más evidente en España con la aprobación de la ley de memoria histórica y con el crecimiento del movimiento de recuperación de la memoria histórica y el recuerdo colectivo de la violencia relacionada con la guerra civil.

En este mismo sentido Páez (2010) afirma que los rituales institucionales de perdón (Apology) y de memoria permiten: asumir los hechos al colectivo (reconocimiento social), incrementar el grado de conductas reparatorias, que se asuma la culpa colectiva, la vergüenza, se refuerce la cohesión social, se asuma el pasado (más allá del simple recuerdo) y haya soporte social para las reparaciones; lo cual favorece la reconciliación intergrupal. Para el autor, las peticiones de perdón públicas van detrás de un reconocimiento público de responsabilidad, lo que implica, una memoria autocrítica, en oposición a una memoria que oculte el pasado (negadora).

Sin embargo, no es posible afirmar que todos las acciones de memorialización tienen efectos positivos en los sujetos y comunidades, ya que algunas de estas, cuando se dan en contextos en los que no hay garantias de no repetición, pueden ser incluso ofensivas para las víctimas; como lo menciona Martín Beristain (2005), quien afirma que estas acciones no protegen contra el duelo alterado, ni son suficientes para la mejora de los sintomas, sobre todo, cuando el contexto social es negativo: o bien porque se mantiene la violencia y la amenaza, o bien porque no hay disposición de la sociedad para acoger, escuchar y validar sus historias y sus reclamos. También se pueden incrementar sintomas como la rumiación, la tristeza, la rabia y el dolor. En el compartir puede haber un revivir las pérdidas y una retroalimentación del dolor que crea un clima emocional negativo. Se supone que la red social está afectada y no hay capacidad de contención (Kunovich \& Hudson, 1999).

Es importante tener en cuenta que el cierre de los procesos de transición puede durar décadas, puesto que son procesos inherentemente contradictorios; por ejemplo Francia con la II guerra mundial, Chile después del arresto de Pinochet, Argentina con la llegada de los Kischner al poder. Estas rupturas y daños no se van a resolver por dos años de una CVR y por un programa de reparaciones. Las heridas quedan, aunque el reconocimiento del pasado en forma de verdad histórica, de confesión y reparación se ha vuelto central y fundamental en la legitimidad del Estado-Nación (Christie, 2007). Los actos simbólicos y las reparaciones son el primer paso para el reconocimiento y la curación de memorias silenciadas; y son factores necesarios (un buen comienzo), pero no son suficientes. De hecho, Jaramillo Marín (2010) sostiene que una de las principales enseñanzas que dejan otros procesos de recuperación del pasado y tramitación de la verdad y la justicia en America Latina y el mundo, es que no basta con que se realicen procesos oficiales de reconstrucción de memoria, para que se sanen las heridas y se dé el 
"nunca más", pues estos procesos son necesarios pero no suficientes, si se quiere la reconciliación.

Es imperativo además que los actos de memoria, para poder ser reparadores, den lugar a la restitución del buen nombre de las personas asesinadas o desaparecidas y de sus familias, pues esta es una forma de reconocer y construir la condición de las víctimas como víctimas y legitimar sus derechos (Arévalo Naranjo, 2010; Estrada, Ripoll \& Rodríguez, 2010). La resolución y la recuperación dependerán también de la forma como cada sujeto personalmente desarrolle su trabajo de trauma y de duelo (proceso para el cual el acompañamiento psicosocial, en el marco de la rehabilitación, puede tener un papel valioso), pero también del contexto, de la idiosincrasia y de la ideologia, de su forma de concebir los derechos humanos y la justicia (Hamber, 1997b; Hamber \& Wilson, 2002; Lykes, Blanche y Hamber, 2003).

Las ideas ilustradas hasta ahora en este apartado, demuestran con claridad la importancia y el valor de las medidas simbólicas y específicamente de la memoria; de todas formas algunas voces como la de Iván Orozco (2009) recomiendan cierta mesura en relación a la justicia transicional, puesto que consideran que ésta es un campo de batalla y negociación que se sitúa en unos delgados hilos entre el deber de memoria que se tiene con las víctimas de la guerra y el derecho a ciertos olvidos en favor de los intereses nacionales, puesto que para este autor, elevados niveles de memoria puede dar lugar a lucha contra la impunidad, pero puede también constituirse en un obstáculo para la transición. Del mismo modo, cuotas altas de olvido hacen imposible la verdad y la justicia; sin embargo, algunos actores sociales e institucionales demandan el cierre de ciertos pasados violentos cuando estos ponen en juego la reconciliación nacional. Al final de cuentas este tema termina siendo un claro dilema al que se enfrentan los Estados, las víctimas los victimarios y la sociedad en general, el cual puede tener importantes implicaciones dentro de los procesos de reparación de las víctimas; por lo que resultará fundamental comprender las construcción de significado de estas últimas al respecto, para que no sean convidadas de piedra, sino protagonistas en los procesos de transción.

\section{Rehabilitación.}

De acuerdo con el ICTJ (2009, p. 40) la rehabilitación incluye la prestación de "atención médica y psicológica y los servicios jurídicos y sociales que requieran las víctimas". De alli que lo planteado hasta el momento ha ido mostrando ciertas indicaciones en lo que respecta a la atención en salud por parte del Estado, la cual constituye un aporte de gran relevancia para esta reflexión sobre la reparación integral, que según diversos investigadores, nacionales e internacionales, debe llevarse a cabo desde una perspectiva psicosocial (Martín Beristain, 2005, 2008, 2010; Hayner, 2008b; Arévalo Naranjo, 2010; Estrada, Ripoll \& Rodríguez, 2010; Rebolledo \& Rondón, 2010; Villa \& González, 2012 y Villa, 2012a, 2012b, 2013) , lo que implica, entre otras cosas, que se desarrolle un proceso complejo, continuo y completo de tal forma que no se reconfiguren escenarios de desesperanza, impotencia y dolor altamente lesivos y revictimizantes que, de ninguna manera, aportan a la reparación integral. 
Adicionalmente se requiere que el acompañamiento psicosocial promueva la transformación de las construcciones de realidad subjetivas, familiares y locales; lo que trasciende las intervenciones clásicas, occidentalizadas y medicalizadas de la psicología que, por centrarse sólo en el psiquismo y en el individuo, resultan insuficientes para este tipo de problemáticas, pues sacan al sujeto de su contexto social, lo que impide hacer una adecuada lectura de los ciclos de la violencia que han existido históricamente en los diversos escenarios en que han tenido lugar las violaciones de los derechos y además, dejan de promover la reconstrucción de los lazos sociales que se fragmentan como consecuencia del conflicto armado (Villa, 2012). La reparación entonces, para ser integral, debe tener lugar en un proceso dialéctico entre lo singular y lo colectivo, el sujeto y la comunidad a la que pertenece.

Henao (2011) formula, en coherencia con lo expresado a lo largo del presente texto, que no es suficiente que se brinde una reparación material a las personas víctimas, sino que además es primordial visualizar la importancia de la salud mental y de los fuertes impactos que ésta recibe en la población víctima del conflicto armado y advierte que:

"Es necesario entender que en la medida en que no se preste la importancia que requiere el tema de salud mental, se presentará con mayor frecuencia la repetición de los hechos: la violencia intrafamiliar, la desintegración de redes sociales de las comunidades, de las familias; el consumo de sustancias psicoactivas, sobre todo en las personas más jóvenes, la ruptura de la rutina académica en la población juvenil; la imperiosa necesidad de mejorar las precarias condiciones básicas de supervivencia por medio del dinero fácil como el narcotráfico y la delincuencia, entre muchas otras consecuencias psicosociales que tiene el conflicto armado" (pág. 69).

Esta afirmación demuestra la relevancia de la atención en salud y dentro de esta del acompañamiento psicosocial; sin embargo, la forma en que se viene desarrollando por el Estado, hasta el momento, contiene dificultades y debilidades sobre las que resulta importante reflexionar. De cara a esto, Arévalo Naranjo (2010) trae a colación el proceso de valoración médica y psicológica emprendido con las víctimas cuya reparación fue ordenada por la Corte Interamericana de Derechos Humanos en las sentencias relativas a los casos de Ituango y Pueblo Bello, Colombia, el cual fue interrumpido por el Gobierno al culminar la primera fase de caracterización, lo que dio lugar a que se presentaran una serie de impactos psicosociales, tales como: pérdida de confianza y desesperanza acompañada por una percepción del Estado como una institución ausente e indiferente frente al compromiso de reparación y a las necesidades y dolor acaecido por las personas víctimas, con lo que en vez de jugar un rol protector, lesiona y revictimiza. A esto se le suma la superposición de lógicas en la realización de los proyectos de atención, donde prima una lógica burocrática y tecnocrática que privilegia el dato, la meta, el producto, sin atender al proceso y a la dinámica de los sujetos en su contexto, y de los contextos habitados por sujetos, seres humanos de carne y hueso (Villa 2013C - conferencia ISEP) 
Otro de los impactos generados por la falta de continuidad en el proceso de acompañamiento psicosocial emprendido con las víctimas antes mencionadas, fue el incremento de la dificultad para elaborar duelos y de la presencia de sentimientos de incertidumbre y otras expresiones de dolor; además de la cristalización de la identidad de víctima- victimizada, que trae consigo efectos emocionales como el embotamiento, la restricción emocional y el aislamiento social (Arévalo Naranjo, 2010). De manera similar Villa (2013a, b) expresa las dificultades que referencian las víctimas cuando la acción psicosocial se centra en visiones médicas o psicologistas que no permiten una mirada integral de los procesos de las víctimas y que en el marco del Estado no se logran ligar de forma asertiva con otros programas de reparación.

De estas y otras experiencias derivan algunas recomendaciones a la hora de efectuar la atención en salud y el acompañamiento psicosocial en el marco de la reparación a las victimas del conflicto armado, entre las que es posible señalar las siguientes: en primer lugar resulta fundamental que exista una coherencia ontológica, metodológica y ético-politica a la hora de realizar las acciones y acompañamientos que sean considerados como psicosociales, lo que hace necesario que, a nivel ontológico, se conciba al ser humano como un sujeto en relación y construcción con otros y otras, a través de procesos de interacción social y simbólica y que se considere la realidad como una construcción social. A nivel epistemológico, es fundamental que no se realice una división o separación tajante entre sujeto cognoscente y objeto conocido, sino que se tenga una mirada centrada en la interacción entre los sujetos que se relacionan en el acto de conocimiento e intervención, lo que implica que quien realice el acompañamiento debe implicarse en las realidades de quien acompaña, es decir, comprender y actuar desde las categorias y marcos simbólicos de este y trascender la visión psicopatológica y epidemiológica para visualizar los procesos sociales de sufrimiento y resiliencia y los significados y sentidos que las personas, familias, grupos y comunidades construyen en torno a sus experiencias, todo esto anclado en unos marcos simbólicos, socio-políticos e históricos (Villa, 2012b; Villa \& Gonzáles, 2012; Blanco, A., \& Valera, S. 2007; Martín-Baró, 1990).

En cuanto al nivel metodológico, se privilegian estrategias de acompañamiento relacionales, es decir, familiares, colectivas, comunitarias y públicas, ya que estas pueden incidir de forma más profunda en los procesos de transformación subjetiva, teniendo en cuenta la naturaleza de las problemáticas que afectan a la población víctima del conflicto armado; no obstante, las acciones individuales no son excluidas. Finalmente, a nivel ético-politico, se tiene una opción fundamental por el otro y por su bienestar, considerando que las diferentes formas de individualización de la responsabilidad e invisibilización del sufrimiento constituyen formas de reproducir el sistema de victimización, por lo que se propende por el cambio social (Villa, 2012b; Villa \& Gonzáles, 2012; Blanco, A., \& Valera, S. 2007; Martín-Baró, 1990).

En segundo lugar, es importante comprender que diversos fenómenos como el daño, el trauma, y el afrontamiento, no son categorias exclusivamente individuales, sino que son procesos biopsicosociales que se inscriben en dinámicas sociales, culturales, políticas e históricas, por lo que se requiere un abordaje complejo y a varios niveles. Esto sugiere que el proceso de reparación no debe centrarse 
exclusivamente en el individuo, ya que con ello se desconocen aspectos relativos a su contexto y a los sentidos sociales que se construyen al interior del mismo. Adicionalmente, es necesario que los profesionales que acompañan los procesos de reparación y específicamente de rehabilitación, tengan un conocimiento de las condiciones del conflicto armado nacional, de los marcos legales y de los sistemas de aplicación de estos (Estrada, Ripoll \& Rodríguez, 2010; Rebolledo \& Rondón, 2010; Villa, 2012b; Villa \& Gonzáles, 2012).

En tercer lugar, se tiene que el objetivo del acompañamiento psicosocial debe apuntar a construir un proceso reflexivo con la población víctima y su red social, lo cual implica considerar la relación que se construye entre quien acompaña y la persona acompañada como un motor que orienta el proceso, en la cual las actitudes de sensibilidad, disposición a prestar apoyo, mantenimiento de la distancia emocional óptima y reconocimiento de las emociones propias y del otro juegan un papel fundamental y en la que se requieren habilidades básicas como la empatía, la contención, la escucha activa y la comunicación afectiva, efectiva y asertiva. Es importante además que se tenga como referente de análisis la identidad de las personas víctimas en relación a su situación actual, a la experiencia violenta sufrida y a las proyecciones a futuro y que se consideren la dignidad y los derechos de las personas atendidas como características inherentes a éstas, cuyo respeto y cumplimiento ha de potenciarse (Arévalo Naranjo, 2010; Estrada, Ripoll \& Rodríguez, 2010; Villa \& Gonzáles, 2012).

Rebolledo \& Rondón (2010) sostienen que si bien en las comunidades existen condiciones de vulneración, no basta con satisfacer las necesidades básicas, sino que también se deben desarrollar acciones que fortalezcan a personas y grupos, amplificando sus recursos culturales y comunitarios, para aportar de forma significativa a la transformación de sus realidades.

En cuarto lugar, se considera que, además de la complementariedad que debe existir entre los diferentes ámbitos de la justicia transicional para que el proceso de reparación sea realmente reparador, es necesario que haya una coordinación y cooperación entre lo psicosocial y lo jurídico, de tal forma que lo psicosocial permita brindar un apoyo a las personas víctimas en torno a la comprensión y el afrontamiento de los procesos jurídicos y los alcances que estos pueden tener en sus vidas, además de tener presentes procesos individuales y colectivos como la identidad, los miedos, los sueños y las percepciones de la realidad a través de un proceso reflexivo, en el que las narrativas emocionales y los procesos de resignificación juegan un lugar esencial (Arévalo Naranjo, 2010; Estrada, Ripoll \& Rodríguez, 2010; Rebolledo \& Rondón, 2010; Villa \& Gonzáles, 2012; Villa, 2012a, 2012b, 2013).

La interdisciplinariedad y la transdisciplinariedad aparecen también como un requisito de gran envergadura a la hora de realizar la atención en salud, pues debido a la complejidad de los daños sufridos por la población víctima se requiere de la acción de médicos (as), enfermeros (as), psiquiatras, psicólogos (as), trabajadores (as) sociales, antropólogos, sociólogos y abogados que tengan clara la perspectiva 
y el enfoque psicosocial de la atención, además de metodologías de intervención que partan desde esta mirada psicosocial. Lo que implica, no solamente, equipos intersectoriales e interinstitucionales, sino también grupos humanos cualificados en esta mirada y esta forma de la acción (Arévalo Naranjo, 2010; Estrada, Ripoll \& Rodríguez, 2010; Rebolledo \& Rondón, 2010; Villa \& Gonzáles, 2012; Villa, 2012a, 2012b, 2013).

En quinto lugar, es importante distinguir entre los tiempos y procesos de reparación jurídica y política y los tiempos psicológicos, pues son de naturaleza diferente y, aunque se impactan mutuamente, no es conveniente creer que son uno sólo y por lo tanto, no es pertinente aplazar el acompañamiento psicosocial hasta adelantar el proceso jurídico, ya que el primero puede facilitar el segundo, debido a que permite que las personas víctimas alcancen un mayor grado de comprensión de su experiencia, lo cual favorece un mayor dominio sobre lo jurídico (Estrada, Ripoll \& Rodríguez, 2010).

En sexto lugar, es relevante reconocer los diferentes lenguajes y formas retóricas que se han instaurado institucional y políticamente, además de identificar los efectos que estos generan en la comprensión que las personas víctimas tienen de su proceso de reparación (Estrada, Ripoll \& Rodríguez, 2010). Al respecto, Gutiérrez de Piñeres Botero, Coronel, \& Pérez (2009) sugieren que el uso de excesivos tecnicismos en el lenguaje constituye otra forma de generar desorientación en las personas víctimas, quienes no alcanzan a comprender claramente la información que se les suministra, lo que puede dar lugar a una victimización secundaria que exacerba el daño y de fondo sugiere una posición epistemológica en la que se concibe al sujeto que se interviene como ajeno, extraño y en la que se marca una línea simbólica (discursiva) que divide y delinea el lugar de experto diferenciado y desconocedor de los marcos, categorias y saberes de quienes atiende (Villa, 2012b).

La evidencia que proveen los hechos antes descritos y las aseveraciones hasta ahoras realizadas exhortan al Estado colombiano a reconocer que aún no cuenta con la capacidad institucional para responder a las demandas de atención en salud desde una perspectiva psicosocial, en el marco de la ejecución de las medidas de reparación a las víctimas del conflicto armado; de tal forma que estas terminan siendo percibidas como paños de agua tibia o paliativos que no transforman la realidad de la gente (Villa Gómez, 2013; Henao, 2011).

Se requiere pues un conocimiento del contexto en el cual se concibe y desarrolla el proceso de reparación, tener una mirada compleja de la situación de la población afectada que apunte hacia la implementación de medidas colectivas, además de incluir la voz de las personas víctimas en las diferentes acciones que se desarrollen (Villa, Tejada, Sánchez \& Téllez, 2007), ya sean jurídicas, psicosociales, de salud fisica, administrativas o simbólicas. Es importante también que, como se mencionó con antelación, se promueva la dignificación, lo que implica que la reparación aparezca como un fenómeno transformador de las condiciones contextuales en las que tuvo lugar la vulneración de los derechos para que estos no vuelvan a repetirse (Arévalo Naranjo, 2010; ICTJ, 2009; Bello\& Millan, 2005). 
Arévalo Naranjo (2010) agrega además que:

"El fortalecimiento en la construcción de significado[de las victimas] sobre el proceso mismo de reparación, y los sentidos que éste encierra para la reconstrucción de sus historias y proyectos de vida, es de vital importancia para conocer qué puede ser útil y qué no dentro del proceso, de tal forma que, aunque se mantengan directrices o principios fundamentales para el desarrollo de las medidas de reparación, no se pueden perder de vista la particularidad y las necesidades de cada persona, familia y comunidad en el proceso" (pág. 38)

El Estado no puede hacerse el de la vista gorda ante las diferentes formas en que las comunidades y los grupos poblacionales se ven impactados por el conflicto armado, despliegan formas de afrontamiento y significan experiencias en función de sus contextos culturales, históricos, sociales y politicos; por lo que para que cualquier intervención sea relevante y efectiva debe atender y comprender estas particularidades y con base en estas construir programas de actuación. El que un acto sea o no reparador depende de la significación que las personas víctimas le adjudiquen al mismo, a la capacidad de integrarlo en sus proyectos de vida y a la relación que cada sujeto y comunidad establezcan entre lo que se les ofrece y lo que han perdido, lo que hace de la reparación una medida intima, singular y simbólica (Beristain, Giorgia, Páez, Pérez, \& Fernández, 1999; Bello \& Millan, 2005; Rebolledo \& Rondón, 2010).

Resulta fundamental que se reconozca el papel activo de las personas víctimas a la hora de significar los procesos de reparación y establecer qué acciones logran resarcir los daños causados por el conflicto y cuáles no, con esto se rompe la lógica de la guerra, reconociendo la autonomía de los sujetos, en aras de lograr una reparación efectiva desde el punto de vista psicosocial (Rebolledo \& Rondón, 2010).

Es importante anotar que una de las dificultades que se han vislumbrado a la hora de presentar propuestas para el acompañamiento psicosocial a las víctimas del conflicto armado en Colombia, ha tenido que ver con que si estas no están en consonancia con los intereses politicos del momento, no son aceptados, por lo que se habla de una polarización de intereses. Por un lado los de las organizaciones del Estado encargadas de ejecutar las medidas de reparación y por otro de organizaciones defensoras y peticionarias de víctimas de la sociedad civil y los grupos académicos que investigan e intervienen en la problemática de las víctimas. De alli la necesidad de crear sinergias entre el Estado, las ONG y la academia, en busca de más y mejores estrategias de acompañamiento al sufrimiento psicológico de las personas como consecuencia de la violencia politica en Colombia (Estrada Mesa, 2010).

\section{Garantias de no repetición.}

Finalmente es importante traer a colación otra temática a la que se le ha ido aportando a lo largo del texto y es el tema de garantias de no repetición. Estas, "consisten en reformas institucionales $y$ en medidas de otra naturaleza que buscan evitar que las 
atrocidades vuelvan a cometerse, y con las cuales se promueve el Estado de derecho $y$ el respeto de los derechos humanos y de los procesos democráticos, se derogan leyes que contribuyen o autorizan las violaciones, se garantiza el control de las fuerzas armadas, de seguridad y de inteligencia, se procura el desmantelamiento de las fuerzas armadas paraestatales y la reintegración social de niños combatientes, entre otros beneficios" (ICTJ, 2009, p. 41)

Al respecto, es importante ilustrar un poco la dinámica nacional. A pesar de las anunciadas desmovilizaciones colectivas e individuales y de los procesos de restituciones de tierras que se han adelantado hasta la fecha, Colombia continúa teniendo un conflicto armado interno, se sigue delinquiendo y la tierra sigue siendo el principal detonante del conflicto; lo que permite afirmar que el país se encuentra en medio de una "guerra sin transición" (Jaramillo Marín, 2010b). Una muestra de ello, son las numerosas persecuciones y revictimizaciones que se han presentado en las zonas donde se ha reclamado la restitución de tierras. Sumado a esto en Colombia continúan los desplazamientos forzados, las amenazas, los asesinatos de líderes y a pesar del proceso de desmovilización que se adelantó en el marco de la Ley de Justicia y Paz, no se ha dado un real desmonte de las estructuras paramilitares sino una mutación de las mismas. Todo esto impide hablar de unas garantías de no repetición (Jaramillo Marín, 2010a, 2010b; González Chaverra, 2010; Villa, 2013).

En relación con lo anterior, Uprimny \& Guzmán (2010), resaltan la importancia de pensar en una reparación transformadora, en donde no se restaure a las víctimas que vivian en situaciones de pobreza, a la misma situación de desigualdad, precariedad y discriminación, sino que se propicie una transformación de esas circunstancias, que posiblemente tuvieron que ver con que se diera el conflicto, pues de no ser así, no se estarian brindando garantias de no repetición y se incurriría en la perpetuación de ciclos interminables de violencia. Se debe entonces pensar la reparación "como una oportunidad de impulsar un mejor futuro" (pág. 253)

Sin embargo, aunque se plantea la importancia de que se den las garantías de no repetición y se hable de generar reparaciones transformadoras como medio para lograrlo, como se mencionó líneas atrás, Chavarría (2012) plantea que, en comparación con la atención prestada a la búsqueda de la verdad, la justicia y a otras medidas de la reparación, es poca la atención que en Colombia se ha brindado al cumplimiento de las garantías de no repetición y a la búsqueda de estrategias para que estas puedan darse realmente.

Otro aspecto que resulta ser de gran importancia para que haya una verdadera transición del conflicto a la paz, es la transformación de los discursos predominantes a nivel nacional, es decir, la transición retórica, que no suele ocurrir de manera suave, sino que históricamente ha implicado la creación de brechas entre los discursos previos y los nuevos, lo que puede generar confusiones a nivel político y en el ámbito de la salud mental. Rebolledo \& Rondón (2010) traen a colación el caso de Sudáfrica para ilustrar cómo el discurso nuevo construido por Nelson Mandela y el Congreso Nacional Africano, cuyo mensaje era la reconciliación y la nueva Sudáfrica, 
constituyó un elemento fundamental para potenciar el proceso de transición. Sin embargo, este caso no es muy común y de hecho en Colombia, uno de los discursos predominantes durante varios años estuvo caracterizado por la paradójica y contradictoria negación de conflicto armado, acompañada simultáneamente por la promoción de un marco jurídico y político de justicia transicional como el de Justicia y Paz, que avanzó entre grandes contradicciones, como la exclusión de algunas actores -"los terroristas"- de los diálogos y la negociación con otros, además de la presentación del imaginario de un Estado sin responsabilidad directa frente a la guerra; esto sumado a la cooptación de la institucionalidad por parte de grupos armados organizados al margen de la ley y la parapolítica, lo que transgrede los derechos de las personas y genera confusiones discursivas que exacerban los daños e impactos generados directamente por el conflicto armado (Estrada Mesa, 2010; Rebolledo \& Rondón, 2010; Jaramillo Marin, 2010b). En un escenario como este, en el que aparecen legitimadas tantas acciones contra la vida y la integridad de las personas, no es posible hablar de condiciones que favorezcan la transición y que por lo tanto promuevan la no repetición de la violencia.

Las posibilidades de una verdadera transición reposan en la intención y el compromiso de todas las partes (no sólo la triada Estado -guerrilla- paramilitares) por elegir formas de relación diferentes a la violencia; sería esto precisamente lo que garantizaría la no repetición. No obstante, esto requiere de un profundo cambio cultural, cambio que es posible pero se teje con el tiempo y con las voluntades, constituyendo un tejido transformador hilado con el dolor como reacción al horror, pero también con la fuerza como opción de cara a la vida.

\section{Conclusiones.}

A partir de lo formulado hasta ahora es posible extraer las siguientes conclusiones, especialmente aterrizadas para el caso de Colombia:

Colombia, debido a sus condiciones contextuales y restricciones institucionales, posee importantes dificultades para hacer realidad la reparación a las personas víctimas, desde los propios marcos de significación de estas últimas, por lo que en numerosas ocasiones ha generado en éstas efectos contrarios a los esperados, revictimizándolas, impactando en su identidad y en el tejido social, con lo que se exacerba el daño e impacto producido por el conflicto armado.

Los significados construidos por la personas víctimas en torno a sus expectativas frente a la verdad, la justicia y la reparación, en sus diferentes mecanismos, difieren en función de sus características socio-culturales, politicas e ideológicas, económicas, étnicas, de género, del ciclo vital, y del tipo de victimización sufrido, por lo que resulta fundamental idear y ejecutar acciones adecuadas y no victimizantes para aproximarse a dichas significaciones en aras de lograr procesos de reparación realmente eficaces y, sobretodo, dignificantes. Es imperativo que dichas acciones obedezcan a una mirada regional y diferencial. 
En medio de un contexto como el colombiano, en el que la mayor parte de la población víctima tiene insatisfechas sus necesidades básicas, el Estado debe trazarse el complicado pero necesario reto de trascender la reparación material, que cuando llega sin ninguna otra medida, puede significar para las personas víctimas un paño de agua tibia o sólo una ayuda, que realmente no aporta a su proceso de recuperación, el cual es complejo y requiere de acciones simbólicas y acompañamiento psicosociales, previo, simultáneo y posterior a la compensación. Además de medidas claras de no repetición y de justicia social que permitan transformar su situación de exclusión y empobrecimiento históricos.

La compensación o indemnización es una medida fundamental dentro del proceso de reparación, pues simbólicamente permite que el Estado reconozca su nivel de responsabilidad; es además una de las medidas que las personas víctimas consideran más significativas pues a través de esta buscan satisfacer algunas de sus necesidades básicas. Sin embargo, la forma en que las compensaciones se han llevado a cabo han impactado negativamente en la identidad, formas de relación, escalas morales de las personas victimas, pues ha estado caracterizada por la inmersión de estas personas en una larga espera y en un marco que según los textos revisados, sigue siendo poco reparador.

Los estudios indican que para las personas víctimas de diferentes naciones, incluida Colombia, la reparación material suele ser considerada como la más importante, lo que puede hallar fundamento en las condiciones de vulnerabilidad en las que estas generalmente se encuentran, en sus múltiples necesidades básicas insatisfechas y en las condiciones estructurales que obstaculizan la transición. En algunas investigaciones aparecen en segundo lugar de relevancia las medidas simbólicas, aunque en algunas naciones estas son incluso más importantes para las víctimas que la indemnización, lo cual obedece a ciertas características diferenciales del conflicto, de la población y de la región en el cual este se desarrolla. En otras investigaciones, la reconciliación, la no repetición y el acompañamiento psicosocial aparecen en segundo lugar, mientras las medidas simbólicas en tercero. Independiente del lugar en el que aparezcan una u otras medidas, el aporte más significativos de los trabajos revisados es el señalamiento que realizan de la necesidad de que haya una complementariedad entre las diversas medidas de reparación y que cuando éstas se efectúen lleven a cabo un reconocimiento público, social y moral de las personas víctimas, lo que resulta ser dignificante.

Resulta fundamental que a la hora de diseñar e implementar las medidas de reparación se tenga como punto de referencia los marcos simbólicos y culturales de las personas víctimas, lo que implica trascender el modelo occidentalizado (centrado en el dato, la burocracia, lo técnico, lo estadístico, el cumplimiento de metas numéricas y la ejecución de un presupuesto), de tal forma que pueda atenderse realmente a las necesidades de la población con la apertura a las complejidades, emergentes y demás situaciones caóticas que tienen lugar en el mundo de la vida y en el mundo social. 
La reconstrucción de la memoria es un proceso complejo, continuo y abierto que suele impactar positivamente la salud mental de los sujetos, familias y comunidades que han sido víctimas, pues posibilita llevar a cabo el cierre de las heridas generadas por el conflicto armado, promover la transformación de las condiciones socio-culturales y politicas y el empoderamiento de las personas y aportar a la reconstrucción del tejido social, al aumento de la cohesión social y al afrontamiento colectivo. No obstante, cuando la ejecución de esta medida se hace desde una lógica racional de los hechos, centrada en las fechas y archivos y no en las historias, en el sufrimiento, en las resistencias y en la dignidad de la gente, puede generar revictimización y generar más daño.

Muchos de los actos simbólicos que han resultado reparadores para los sujetos, familias y comunidades que han sido víctimas de la violencia politica han surgido como iniciativa de estos mismos, expresados por medio de sus rituales tradicionales o de rituales creados (de manera performativa), lo que muestra que la gente tiene sus propios recursos para hacer frente al dolor y a las heridas y que estos recursos deben ser escuchados, validados e incluidos en la propuesta de reparación que realiza el Estado, para que realmente logre impactar significativamente en las personas. Esto requiere que se realicen transformaciones ontológicas y epistemológicas profundas en la forma en que las instituciones estatales se aproximan a la realidad de las personas víctimas.

El Estado colombiano tiene aún mucho por aprender en materia de rehabilitación, pues a través de la ejecución de esta medida ha ocasionado más daño en la población víctima. Se requiere entonces que la atención en salud se lleve a cabo desde una perspectiva psicosocial, lo que implica una coherencia ontológica, epistemológica, metodológica y ética-política, que se desarrolle a través de un proceso continuo y complejo que promueva la transformación de las construcciones de realidad, que trascienda la visión occidentalizada y medicalizada de los fenómenos humanos y que se realicen intervenciones interdisciplinares, transdisciplinares, intersectoriales e interinstitucionales que obedezcan a un profundo conocimiento de la historia del conflicto, del contexto y de los significados que las personas víctimas han construido

Las condiciones sociopolíticas y culturales colombianas están caracterizadas por la continuidad del conflicto armado y de la violación de los derechos humanos, por la mutación de las estructuras armadas desmovilizadas, por la dominancia de discursos contradictorios respecto a la transición y la subyacente confusión política y mental que estos generan, por la pervivencia de muchas de las personas víctimas en situaciones de vulnerabilidad e insatisfacción de sus necesidades básicas y por las pocas reformas institucionales. Todo estas condiciones impiden culminar con la cadena violenta que por años ha acaecido el país y por lo tanto hacen de las garantías de no repetición una realidad aún inalcanzable. 


\section{Referencias bibliográficas.}

Agencia Nacional para la Superación del Pobreza Extrema (ANSPE). (2013). Enfoque Diferencial: Dirección Gneral. Colombia.

Aguilar, P., Balcells, L., \& Cebolla, H. (2011). Actitudes de los españoles ante las medidas de justicia transicional relativas a la guerra civil y al franquismo. Revista Internacional de Sociología, 69(1), 59-90.

Arévalo Naranjo, L. (2010). Atención y reparación psicosocial en contextos de violencia sociopolitica: una mirada reflexiva. Revista de estudios sociales(36), 29-39.

Baró, I. M. (1990). Piscología Social de la Guerra: Trauma y Terapia. El Salvador: UCA Editores.

Bello, M. N., \& Millan, C. (2005). La intervención institucional en contextos culturalmente distintos: lógicas en tensión y contradicción. FACULTAD DE CIENCIA HUMANAS, UNIVERSIDAD NACIONAL DE COLOMBIA.

Beristain, C. M. (2009). Diálogos sobre la reparación: que reparar en los casos de violaciones de derechos humanos. Quito: Ministerio de Justicia y Derechos Humanos.

Beristain, C. M. (2010). El derecho a la reparación en los conflictos socioambientales. . Bilbao: Hegoa y Universidad del País Vasco.

Beristain, C. M., Dona, G., Paéz, D., Sales, P., Pau, \& Fernández, I. (1999). Recosntruir el tejido social: Un enfoque crítico de la ayuda humanitaria. Barcelona: Icaria editorial/ Antrazyt.

Beristain, C. M., Páez, D., Rimé, B., \& Kanyangara, P. (2010). Efectos psicosociales de la participación en rituales de justicia transicional. Revista de Psicologia, 28(1), 9- 35.

Beristain, M. (2011). Transformar las sociedades después de la violencia política. Verdad, reconciliación y salud mental. Barcelona: Ediciones Bellaterra.

Blanco, A. \&. (s.f.). La razón compasiva como acción ideológica: una nueva mirada a la psicología de la liberación .

Blanco, A., \& Valera, S. (2007). Los fundamentos de la intervención psicosocial. En A. Blanco, \& J. Rodríguez Marín, Intervención psicosocial (págs. 3-44). España: Pearson.

Carvacho, H., Manzi, J., Haye, A., González, R., \& Cornejo, M. (2013). Consenso y Disenso en la Memoria Histórica y en las Actitudes Hacia la Reparación en Tres Generaciones de Chilenos. Psykhe, 22(2), 33-47. 
Casas casas, A., \& Herrera Tolaza, G. (2008). El juego político de las reparaciones: un marco analítico de las reparaciones en procesos de jsticia transicional. Papel Político, 197-223.

Chavarría Olarte, G. C. (2012). Estrategias utilizadas para la satisfacción de las garantías de no repetición en desmovilizados de grupos armados ilegales: un estudio con desmovilizados de grupos paramilutares de las Autodefensas Unidas de Colombia. Medellín, Valle de Aburrá- Colombia. Facultad de Derecho y Ciencias Politicas, 42(116), 195-252.

CNNR. (2007). Comisión Nacional de Reparación y Reconcilización: Recomendación de criterior de reparación y proporcionalidad restaurativa. Bogotá.

COALICO \& Grupo de Trabajo pro Reparación Integral. (2008). Reparación Integral para Niños y Niñas Víctimas del Conflicto Armado. Colombia: Corporación AVRE.

Congreso de Colombia. (2005). Ley 975 de 2005.

Corporación Humanas; Centro Regional de Derechos Humanos y Violencia de Género \& Galvis, María Clara. (2008). Situación en Colombia de la Violencia Sexual contra las Mujeres. . Colombia: Contra la Violencia Sexual.

Corte Constitucional. (2008). Auto 092. Colombia.

Estrada Mesa, Á. M. (2010). Impacto de la dináica política colombiana en los procesos de reparacióna las víctimas de la violencia poklítica. Recista de Estudios Sociales(36), 133-144.

Espinosa, N., Rosero, A. G., Bastidas, W., \& Rojas, J. E. M. (2011). Estado actual de los derechos a la identidad, a la vida y la vivienda en la población víctima del desplazamiento forzado en Antioquia. El Ágora USB, 11(2), 253-278.

Espinoza Menendez, N., Rosero, A., Bastidas Lopera, W., \& Monsalve Rojas, J. E. (2011). Estado actual de los derechos a la identidad, a la vida y la vivienda en la población víctima del desplazamiento forzado en Antioquia; Current state of the rights to identity, to life, and to housing, in the population victim of forced displacement in Antioquia. Agora USB, 11(2), 253-278.

Espinosa Menendez, N., Galindo Rosero, A., Bastidas Lopera, W., \& Monsalve Rojas, J. E. (2012). Estado actual de la población víctima de desplazamiento forzado en cuanto al enfoque diferencial de su atención, el derecho a la integridad, la subsistencia mínima y al ingreso económico. Agora USB, 12(1), 19-46.

Estrada Mesa, Á. M., Ripoll Núñez, K., \& Rodríguez Charry, D. (2010). Intervención psicosocial con fines de reparación con víctimas y sus familias afectadas por el conflicto armado interno en Colombia:equipos psicosociales en contextos jurídicos. Revista de Estudios Sociale(36), 103-112.

Fuenzalida, P. (2007). Las políticas de reparación en Chile y sus consecuencias en los hijos de los detenidos - desaparecidos. 1990-2000. Chile: Universidad de Estocolmo. 
Gaborit, M. (2006). Memoria Histórica:Relato de las víctimas. Pensamiento Psicológico, 2(6).

Girón Ortiz, Claudia; Puerto Barrera, Betty \& Fundación Manuel Cepeda Vargas. (2006). Módulo de la dimensión simbólica y cultural de la reparación integral. Colombia: Grupo de trabajo pro reparación integral.

Gómez Cordoba, Oscar; Corporación AVRE Apoyo a víctimas de violencia sociopolítica prorecuperación emcoional. (2006). Aspectos psicosociales de la reparación integral. Colombia.

González Chavarría, A. (2010). Justicia transicional y reparación a las víctimas en Colombia. Revista Mexicana de Sociologia, 72(4), 629-658.

Grieff, P. (2011). Algunas reflexiones acerca del desarrollo de la Justicia Transicional. Anuario de Derechos Humanos(7), 17-39.

Grupo de Memoria Histórica. (2013). ¡Basta ya! Colombia: Memorias de guerra y dignidad. Colombia : Centro Nacional de Memoria Histórica.

Gutiérrez Agüello, Soraya; Pacheco García, Lucia \& Corporación Colectivo de Abogados José Alvear Restrepo . (2006). La reparación integral un derecho de las víctimas. Grupo de trabajo pro Reparación Integral.

Gutiérrez de Piñeres Botero, C., Coronel, E., \& Pérez, C. A. (2009). Revisión teórica del concepto de victimización secundaria. Liberabit. Revista de Psicologia,, 5(1), 49-58.

Henao, M. C. (2011). Atención en salud mental a víctimas del conflicto armado colombiano. Rostros \& Rastros: Justicia, comunidad y responsabilidad social(6), 67-70.

Ibarra Melo, M. E. (2011). Mujeres, verdad, justicia y reparación en Colombia. Universitas Humanística(72), 247-273.

ICTJ; DeJusticia. (2009). Reparar en Colombia: los dilemas en contextos de conflicto, pobleza y exclusión. Colombia.

Insuasty Rodriguez, A., Balbin, J., Bastidas, W., Carrión Suarez, J., Pineda, J. E., \& Mejía, W. (2010). Las victimas en contextos de violencia e impunidad: caso Medellin. MISC.

Isaacs, A. (2006). ¿Superando el pasado?: verdad, justicia y resarcimiento en Guatemala. Área América Larina(1).

Jaramillo Marín, J. (2010a). Reflexiones sobre los usos y abuso de la verdad, la justicia y la reparación, en el proceso de Justicia y Paz Colombiano (2005-2010). Ciencia Politca, 15(1), 13-46. 
Jaramillo Marín, J. (2010b). Narrando el dolor y luchando contra el olvido en Colombia. Recuperación y trámite institucional de las heridas de la guerra. Revista Sociedad y Economia(19), 205- 228.

Jaramillo Marín, J. (2011). Expertos y comisiones de estudio sobre la violencia en Colombia. Estudios Políticos(39), 231-258.

Jaramillo Marín, J. (2011). Justicia Transicional en tiepo del deber de memoria (Reseña). Revista Estudios Sociales, 5(1), 158-163.

Laplante, L. (2007). Después de la verdad: Demandas para reparación en el Perú postcomisión de la verdad y reconciliación. Antípoda(4).

Lira, E. (2010). Taruma, duelo, reparación y memoria. Revista de Estudios Sociales(36), 14-28.

Lira, E., \& Loveman, B. (2005). Políticas de reparación: 1990-2004. Santiago: LOM Ediciones.

Lozano, J. J., \& Morris, H. (2010). Impunity. Recuperado el 9 de Marzo de 2014, de Youtube: http://www.youtube.com/watch?v=BjDzVxY4mhg

Marin Ortiz, I., \& Gaviria Betancur, P. (2010). Elementos para una politica de verdad, justicia y reparación integral para las víctimas. En E. Kiza, \& A. Rettberg, Reparación en Colombia: ¿Qué quieren las víctimas?: Retos, desafios y alternativas para alcanzar la integridad (págs. 79-106). Colombia: Deutsche Gesellschaft für; Technische Zusammenarbeit (GTZ); GmbH; Cooperación Técnica Alemana; ProFis.

Ministerio del Interior. (2012). Ley de Víctimas y Restitución de Tierras y sus Derechos Reglamentarios. Bogotá, Cundinamarca, Colombia. Obtenido de Ley de Víctimas y Restitución de Tierras y sus Derechos Reglamentarios: http:// www.leydevictimas.gov.co/documents/10179/19132/completo.pdf

Molina Valencia, N. (2010). Reconstrucción de memoria en historias de vida: Efectos políticos y terapéuticos. Revista de Estudios Sociales(36), 64-75.

ONU, Comisión de Derechos Humanos. (2005). Principios y directrices básicos sobre el derecho de las víctimas de violaciones manifiestas de las normas internacionales de derechos humanos y de violaciones graves del derecho internacional humanitario a interponer recursos y obtener reparaciones.

Pinto Velásquez, E. (2011). Que canten las gallinas, no sólo el gallo: memoria, mujeres y tierra. Trabajo Social, 13, 43-59.

Piper, I., Fernández, R., \& Íñiguez, L. (2013). Psicología Social de la Memoria: Espacios y Politicas del Recuerdo. Psykhe, 22(2), 19-31.

Rebolledo, O., \& Rondón, L. (2010). Reflexiones y aproximaciones al trabajo psicosocial con víctimas individuales y colectivas en el marco del proceso de reparación. Revista de Estudios Sociales(36), 40-50. 
Rettberg, A. (2008). Reparación en Colombia ¿Qué quieren la víctimas? Colombia: Deutsche Gesellschaft fur Technische Zusammenarbeit (GTZ) GmbH.

Rhone, H. C. (2006). Victims' Perspectives in Intercultural Comparison: Victims' Perspectives in Intercultural Comparison. Max Planch Institut fürausländiches und internationales Strafrecht .

Segal, H. (1973). Introduction to the work of Melanie Klein. Great Britain: Karnac Books.

Uprimny Yepes, R., \& Guzmán Rodríguez, D. E. (2010). En búsqueda de un concepto transformador y participativo para las reparaciones en contextos transicionales. Revista Colombiana de Derecho Internacional(17), 231-286.

Villa Gómez, J. D. (2013). Consecuencias Psicosociales de la participación en escenarios de justicia transicional en un contexto d econflicto, impunidad y notransición. Agora, 13(2), 307-338.

Villa, J. D. (2012). La Acción y el Enfoque Psicosocial de la Intervención en Contextos Sociales: ¿Podemos pasar de la moda a la precisión teórica, epistemológica y metodológica? AGORA, 349-365.

Villa, J. D., \& Gonzáles, P. A. (2012). Elementos para la intervención psicosocial y en la salud mental a víctimas de violencia politica en Antioqua. Medellín: Gobernación de Antioquia.

\section{Nota:}

\footnotetext{
${ }^{1}$ Esta revisión bibliográfica y documental hace parte de la investigación: "Construcción de significados sobre la reparación institucional y la reparación desde abajo de víctimas del conflicto armado en la ciudad de Medellín y el Municipio de San Carlos", y pretendemos acercarnos a las dinámicas, respuestas, miradas y procesos que se han suscitado alrededor de la reparación en el mundo; con el fin de establecer diálogos, cuestionamientos, comparaciones y recomendaciones a los procesos que se abordan en el territorio colombiano.
} 